\title{
Recent Development of the Theory of Completely Bounded Maps between $C^{*}$-Algebras
}

By

\author{
Jun TOMIYAMA*
}

\begin{abstract}
Recent development of the theory of completely bounded maps between $C^{*}$-algebras such as those results by Wittstock, Smith, Paulsen and Huruya are reviewed and discussed as well as the author's results.
\end{abstract}

In recent development of the theory of operator algebras it has been recognized that particularity of infinite dimensional noncommutative order structure is in their matricial order and appropriate positivity of linear maps compatible with this order structure should be complete positivity. Thus as counterparts of completely positive maps we have to be naturally concerned with completely bounded maps when we consider bounded linear maps on operator algebras closely related to their structure. Recent results such as Christensen [5] and Haagerup [6] give evidence of this fact in the sense that solutions of the problems are equivalent to complete boundedness of involved maps.

In this paper we intend to review recent development of the basic theory of completely bounded maps on $C^{*}$-algebras. We shall be however mainly concerned with the results for the class of all completely bounded maps. In Section 2 we describe the solution on a pair of $C^{*}$-algebras between which every bounded map becomes completely bounded. Results in this section also mean that in most cases the subspace of completely bounded maps is quite thin in the whole space of bounded linear maps. We shall deal with the growth of the norms of multiplicity maps in Section 3, determining the case where the norms of $n$ multiplicity map $\tau_{n}$ always coincides with the completely bounded norm $\|\tau\|_{c b}$. In this section Theorem 3.2 and Proposition 3.4 seem to be new. Section 4 is

Received January 5, 1983.

* Faculty of Science, Niigata University, Niigata 950-21, Japan. 
devoted to the discussions surrounding recent Wittstock's theorem [25] about completely positive decompositions of completely bounded maps with related problems. The last section is concerned to introduce other topics about completely bounded maps.

\section{§1. Preliminaries}

Throughout this paper by $M_{n}$ we mean the $n \times n$ matrix algebra over the complex numbers and by $B(H)$ the algebra of all bounded linear operators on a Hilbert space $H$. The $n \times n$ matrix $C^{*}$-algebra over a $C^{*}$-algebra $A$ is written by $M_{n}(A)$. When $A$ is the algebra $M_{k}$, the algebra $M_{n}\left(M_{k}\right)$ is also regarded as the matrix algebra $M_{k}\left(M_{n}\right)$ over $M_{n}$ and moreover we sometimes consider $M_{n}\left(M_{k}\right)$ as the $C^{*}$-tensor product $M_{n} \otimes M_{k}$. We write generally as $A \otimes B$ the minimal $C^{*}$-tensor product of two $C^{*}$-algebras $A$ and $B$.

Let $\tau$ be a linear map from a $C^{*}$-algebra $A$ to a $C^{*}$-algebra $B$. The map $\tau$ is said to be completely bounded if the multiplicity map:

$$
\tau_{n}:\left[a_{i j}\right] \in M_{n}(A) \longrightarrow\left[\tau\left(a_{i j}\right)\right] \in M_{n}(B)
$$

(sometimes written as $\tau \otimes 1_{n}$ to emphasize the order of the multiplicity) is bounded uniformly with respect to $n$. In this case we call the norm $\|\tau\|_{c b}=\sup \left\|\tau_{n}\right\|$ the completely bounded norm of $\tau$. On the other hand if $\tau_{n}$ is positive for every $n$ we call $\tau$ a completely positive map. A completely positive map is necessarily completely bounded with $\|\tau\|_{c b}=\|\tau\|(=\|\tau(1)\|$ if $A$ is unital (cf. [2])).

For the definition of completely bounded maps it will be worth to notice here that one can employ the following another definition. Namely:

A map $\tau$ is completely bounded if and only if for an infinite dimensional Hilbert space $H$ there exists a map $\tau_{\infty}$ from the $C^{*}$-tensor product $A \otimes B(H)$ (or $A \otimes K(H)$ for the algebra of all compact operators on $H$ ) to $B \otimes B(H)$ (resp. $B \otimes K(H))$ such that $\tau_{\infty}(a \otimes b)=\tau(a) \otimes b$ with $\left\|\tau_{\infty}\right\|=\|\tau\|_{c b}$.

In fact, the existence of such a map from usual definition of complete boundedness is a consequence of the following estimate. Namely let $e$ be an $n$-dimensional projection in $H$. We have then for every element $\sum_{i=1}^{k} a_{i} \otimes b_{i}$ in $A \otimes B(H)$ (or $A \otimes K(H)$ )

$$
\left\|\sum_{i=1}^{k} \tau\left(a_{i}\right) \otimes e b_{i} e\right\| \leq\left\|\tau_{n}\right\|\left\|\sum_{i=1}^{k} a_{i} \otimes e b_{i} e\right\| \leq\|\tau\|_{c b}\left\|\sum_{i=1}^{k} a_{i} \otimes e b_{i} e\right\|,
$$


which implies that

$$
\left\|\sum_{i=1}^{k} \tau\left(a_{i}\right) \otimes b_{i}\right\| \leq\|\tau\|_{c b}\left\|\sum_{i=1}^{k} a_{i} \otimes b_{i}\right\|
$$

For standard results of the theory of $C^{*}$-algebras, we refer mainly the book of Takesaki [17].

\section{§2. Difference between Bounded Linear Maps and Completely Bounded Maps}

In the theory of operator algebras, completely positive maps and derivations in a $C^{*}$-algebra are good examples of completely bounded maps. On the other hand one finds in literature ([13], [22], etc.) examples of bounded maps on $C^{*}$. algebras which are not completely bounded. It seems however that among others the following example is quite simple and useful. Let the algebra $B(H)$ regard as the algebra of matrices of infinite order with respect to a fixed complete orthonormal basis in $H$. The transpose map $\theta(\infty)$ of those matrices then becomes a unital positive map in $B(H)$. Moreover it is known that $\left\|\theta(\infty)_{n}\right\|=n$ for every $n$, hence the map is not completely bounded (cf. [21]). This result is a simple consequence of well used trick about matrix units system of minimal projections of $B(H)$ with respect to the fixed basis in $H$. Indeed the norm of the block matrix $\left[e_{i j}\right](i, j=1,2,3, \ldots, n)$ for such a system is $n$, whereas the norm of the matrix $\left[e_{j i}\right]$ is one. The argument is also applied to the transpose map $\theta(n)$ in $M_{n}$ to show that the norms of the maps $\theta(n)_{k}^{\prime} s$ increase as $1,2, \ldots$ up to $n=\|\theta(n)\|_{c b}$.

Let $A$ and $B$ be $C^{*}$-algebras. We denote by $L(A, B)$ the space of all bounded linear maps from $A$ to $B$ and by $C B(A, B)$ the space of all completely bounded maps from $A$ to $B$ with sometimes completely bounded norm. We note that the space $C B(A, B)$ is complete with respect to the completely bounded norm. In particular we denote $L(A)=L(A, A)$ and $C B(A)=C B(A, A)$.

It has been known that if the range algebra $B$ is either commutative or $M_{n}$ then $C B(A, B)=L(A, B)$ for every $C^{*}$-algebra $A$. Furthermore the result holds when $B$ is a subhomogeneous $C^{*}$-algebra and $A$ is any $C^{*}$-algebra. We recall that a $C^{*}$-algebra is said to be subhomogeneous with bounded degree $n$ if every irreducible representation is finite dimensional with dimension not greater than $n$ or equivalently if $B$ is a $C^{*}$-subalgebra of $M_{n}(C)$ for some commutative $C^{*}$-algebra $C$. The converse of the above statement is also known to be valid ([12]). On the other hand, Loebl also proved in [12] that the equality 
$C B(A, B)=L(A, B)$ holds for any $C^{*}$-algebra $B$ if and only if $A$ is finite dimensional. In these circumstances, the following characterization by Huruya and the author in [8: Corollary 4] has been expected for years. The same result is also proved by Smith (see Theorem 2.4 below) in a slightly different formulation.

Theorem 2.1. Let $A$ and $B$ be $C^{*}$-algebras. Then the following assertions are equivalent:

(a) Every bounded linear map from $A$ to $B$ is completely bounded, i.e. $C B(A, B)=L(A, B)$.

(b) Either $A$ is finite dimensional or $B$ is subhomogeneous.

In order to illustrate a method of our discussions throughout this paper we shall follow the steps of the implication (a) $\Rightarrow(b)$. The first step is based on the following result, the idea of which is originally due to Lanford's example found in [13], [22].

Lemma 2.2. Let $l^{\infty}(n)$ be the $C^{*}$-algebra of finite sequences on $k=1,2 \ldots$, $n$. For the integer $m=2^{n}$, there exists then a linear map $\tau$ of $l^{\infty}(n)$ to $M_{m}$ such that $\|\tau\| \leq 1$ and $\left\|\tau \otimes 1_{m}\right\| \geq \sqrt{n / 2}$, i.e. $\|\tau\|_{c b} \geq \sqrt{n / 2}$.

The number $\sqrt{n / 2}$ is a little optional and may be adjusted in a certain way (cf. [13: p. 125]) as well as the definition of the map.

Let $A$ be an infinite dimensional $C^{*}$-algebra, then it contains a sequence of commuting orthogonal positive elements $\left\{a_{i}\right\}$ of norm one. Let $\left\{\varphi_{i}\right\}$ be a sequence of states on $A$ such that $\varphi_{i}\left(a_{j}\right)=\delta_{i j}$. The $C^{*}$-subalgebra $C$ generated by any $n$-elements $\left\{a_{i_{1}}, a_{i_{2}}, \ldots, a_{i_{n}}\right\}$ can be identified with the algebra $l^{\infty}(n)$ and by a projection of norm one from $A$ to $C$ we can define a map: $A \rightarrow C \cong l^{\infty}(n)$ $\rightarrow M_{2^{n}}$ with the same property as in Lemma 2.2. Moreover the map can be defined so as to vanish on the $C^{*}$-algebra generated by other elements $\left\{a_{i} \mid i \neq\right.$ $\left.i_{1}, i_{2}, \ldots, i_{n}\right\}$.

Following Smith [16] we write

$$
k_{n}(A)=\sup \left\{\|\tau\|_{c b} \mid \tau: A \longrightarrow M_{n},\|\tau\| \leq 1\right\} .
$$

This index shows main feature of the present discussion. For instance, the above argument leads to the conclusion that $\lim _{n \rightarrow \infty} k_{n}(A)=\infty$ if $A$ is infinite dimensional, particularly if $A=l^{\infty}$.

For the second step of the proof we need the following approximate factorization maps of the identity map of $M_{n}$ through a $C^{*}$-algebra $B$ (nonunital version 
of [16: Lemma 2.7]).

Lemma 2.3. Suppose that a $C^{*}$-algebra $B$ has an irreducible representation with dimension not less that $n$. Then for positive number $\varepsilon$ there exist factorization maps

$$
M_{n} \stackrel{\phi}{\longrightarrow} B \stackrel{\psi}{\longrightarrow} M_{n}
$$

of contractive completely positive maps $\phi$ and $\psi$ such that $\|\psi \circ \phi-\mathrm{id}\|<\varepsilon$.

An exact factorization, that is, $\psi \circ \phi=$ identity in $M_{n}$ is also obtained by the author in [20: Lemma 1.1] under the same assumption for $B$ but in more general formulations. The result is useful for arguments of completely positive maps, but the latter is somewhat restrictive to use in the present situation because in this case the map $\phi$ may not be assured to be contractive in general.

Now suppose further that $B$ has irreducible representations of arbitrarily high dimensions. With help of the above lemmas one can find a family of completely bounded maps $\left\{\tau^{n} \mid n=1,2 \ldots,\right\}$,

$$
\tau^{n}: A \longrightarrow C^{*}\left(a_{i} \mid i=1,2, \ldots\right) \longrightarrow l^{\infty}(n) \longrightarrow M_{2^{n}} \longrightarrow B
$$

such that $\left\|\tau^{n}\right\|_{c b}$ is not less than arbitrarily close number to $\sqrt{n / 2}$ and the family of maps $\left\{\tau^{n} \mid C^{*}\left(a_{i} \mid i=1,2, \ldots\right)\right\}$ have disjoint supports. We can then prove that a suitable weighted lacunaly sum of these maps keeps its range in $B$ as a bounded map and it is not completely bounded. Thus in this case the subspace $C B(A, B)$ is not closed in $L(A, B)$ in the uniform norm. On the other hand, each map $\tau^{n}$ is obviously a compact map so that the resulting map is also compact. Let $K(A, B)$ be the space of all compact linear maps from $A$ to $B$. The above observation leads us to another aspects of the case $C B(A, B)=L(A, B)$. In fact, we have:

Theorem 2.4. The following assertions are equivalent:

(a) $C B(A, B)=L(A, B)$.

(c) $C B(A, B)$ is closed in $L(A, B)$ with respect to the uniform norm.

(d) $K(A, B) \subseteq C B(A, B)$.

(e) Every compact map from $A$ to $B$ is a limit of maps of finite rank with respect to the completely bounded norm.

When $B$ is subhomogeneous, the assertion (a) $\Rightarrow(e)$ follows from the fact that $B$ satisfies the metrical approximation property by [4]. Note that in this case the completely bounded norm is equivalent to the uniform norm. 
The condition (c) is due to Smith [16: Theorem 2.8] and other conditions are proved by Huruya in [9: Theorem 5]. Moreover the following application of the Theorem is given in [9].

Proposition 2.5. For two $C^{*}$-algebras $A$ and $B$ the greatest crossnorm $\gamma$ is equivalent to the maximal $C^{*}$-crossnorm if and only if either $A$ or $B$ is finite dimensional.

Indeed a point of the proof in [9] is to show that if $B$ is not subhomogeneous every bounded linear map on $A$ becomes completely bounded because of the assumption of the Proposition.

The case where the minimal $C^{*}$-crossnorm is equivalent to the least crossnorm $\lambda$ is determined by Wassermann [25] (The condition is that either $A$ or $B$ is subhomogeneous).

Now except the above extremal case the subspace $C B(A, B)$ seems to be quite thin in $L(A, B)$. In fact, the following result is proved in [16: Theorem 2.5].

Theorem 2.6. Let $A$ be an infinite dimensional $C^{*}$-algebra and $B$ be $a C^{*}$ algebra containing the subalgebra $\sum_{n=1}^{\infty} \oplus M_{n}$ (for instance any properly infinite von Neumann algebra), then the subspace $C B(A, B)$ is nowhere dense in $L(A, B)$.

Since a subspace with nonempty interior coincides with the whole space, in order to see the Theorem it suffices to show that $C B(A, B)$ is not dense in $L(A, B)$. This will be done again by the method of factorization maps through $l^{\infty}(n)$ and $M_{n}$, finding a map in the interior of the complement of $C B(A, B)$.

There is a conjecture by Smith that the subspace $C B(A, B)$ would be always nowhere dense in $L(A, B)$ if $A$ is infinite dimensional and $B$ is not subhomogeneous.

\section{§3. Growth Conditions of the Norms of the Multiplicity Maps}

The problem of the growth of the norms of the multiplicity maps is the problem mainly concerned with the range algebras. In fact the following early results of Loebl [12] and Ando [1] show a big contrast between the case of range algebras and the case of domain algebras.

Proposition 3.1. (a) Let $B$ be a $C^{*}$-algebra. Then every bounded linear 
map $\tau$ from an arbitrary $C^{*}$-algebra $A$ to $B$ is completely bounded and $\|\tau\|=$ $\|\tau\|_{c b}$ if and only if $B$ is commutative.

(b) Let $A$ be a $C^{*}$-algebra. Then every bounded linear map from $A$ to any $C^{*}$-algebra $B$ is completely bounded and $\|\tau\|=\|\tau\|_{c b}$ if and only if $A$ is commutative and at most two dimensional.

In general a weighted direct sum of those transpose maps $\theta(n)$ of $M_{n}$ shows that there is a completely bounded map the norms of whose multiplicity maps are strictly increasing. There is however a case in which growth of those norms of the multiplicity maps of each map stops at the stage of a fixed $n$. The following theorem partly characterizes this case. This kind of result seems to be important for further analysis of matricial structure of operator algebras.

Theorem 3.2. Let $A$ and $B$ be $C^{*}$-algebras and let $n$ be a fixed positive integer. Consider the following assertions.

(a) Every completely bounded map $\tau$ from $A$ to $B$ attains the norm $\|\tau\|_{c b}$ at $\tau_{n}$, i.e. $\left\|\tau_{n}\right\|=\|\tau\|_{c b}$.

(b) For every completely bounded map $\tau$ from $A$ to $B$ we have that $\left\|\tau_{n}\right\|=$ $\left\|\tau_{n+1}\right\|$.

(c) Either $A$ or $B$ is subhomogeneous with bounded degree $n$.

Then $(\mathrm{a}) \Rightarrow(\mathrm{b}) \Rightarrow(\mathrm{c})$ and if $B$ is subhomogeneous with bounded degree $n$, every bounded (necessarily completely bounded) linear map from any $C^{*}$ algebra $A$ to $B$ attains its completely bounded norm at $\tau_{n}$.

Proof. (b) $\Rightarrow$ (c). Suppose that both algebras $A$ and $B$ have irreducible representations whose dimensions are greater than $n$. We shall show that there exists a completely bounded map $\tau$ of $A$ to $B$ such that $\|\tau\|_{n} \subsetneq\left\|\tau_{n+1}\right\|$. Let $\phi$ and $\psi$ be completely positive contractions in Lemma 2.3 between $M_{n+1}$ and $B$ for a positive number $\varepsilon_{1}$ with $(n+1)^{2} \varepsilon_{1}<1$. We consider first the map $\tau^{\prime}=\phi \circ \theta(n+1)$ from $M_{n+1}$ to $B$. Then

$$
\begin{aligned}
\left\|\tau_{n+1}^{\prime}\right\| & \geq\left\|\psi_{n+1} \circ \phi_{n+1} \circ \theta(n+1)_{n+1}\right\| \\
& \geq\left\|\theta(n+1)_{n+1}\right\|-\left\|\left(\psi \circ \phi-\mathrm{id}_{n+1}\right) \otimes 1_{n+1} \circ \theta(n+1)_{n+1}\right\| \\
& \geq n+1-(n+1)^{2} \varepsilon_{1}>n \geq\left\|\tau_{n}^{\prime}\right\| .
\end{aligned}
$$

The third inequality follows from the estimate that $\left\|(\psi \circ \phi-\mathrm{id}) \otimes 1_{n+1}\right\|<(n+1) \varepsilon_{1}$. Now take an element $x$ of $M_{n+1}\left(M_{n+1}\right)$ such that $\|x\| \leq 1$ and $\left\|\tau_{n+1}^{\prime}(x)\right\|>$ $\left\|\tau_{n}^{\prime}\right\|$. We choose next a pair of completely positive contractions $\sigma$ and $\rho$ in Lemma 2.3 between $M_{n+1}$ and $A$ for an $\varepsilon_{2}>0$ with $(n+1)\left\|\tau_{n+1}^{\prime}\right\| \varepsilon_{2}<\left\|\tau_{n+1}^{\prime}(x)\right\|-$ 
$\left\|\tau_{n}^{\prime}\right\|$. We assert that the completely bounded map $\tau=\tau^{\prime} \cdot \rho$ satisfies the condition. In fact put $y=\sigma_{n+1}(x)$ in $M_{n+1}(A)$ then $\|y\| \leq 1$ and we have

$$
\begin{aligned}
\left\|\tau_{n+1}^{\prime}(x)\right\| & =\left\|\tau_{n+1}^{\prime}\left(x-\rho_{n+1} \circ \sigma_{n+1}(x)+\rho_{n+1}(y)\right)\right\| \\
& \leq\left\|\tau_{n+1}(y)\right\|+\left\|\tau_{n+1}^{\prime}\left(\rho \circ \sigma-\mathrm{id}_{n+1}\right) \otimes 1_{n+1}(x)\right\| \\
& \leq\left\|\tau_{n+1}(y)\right\|+\left\|\tau_{n+1}^{\prime}\right\|(n+1) \varepsilon_{2} \\
& <\left\|\tau_{n+1}(y)\right\|+\left\|\tau_{n+1}^{\prime}(x)\right\|-\left\|\tau_{n}^{\prime}\right\| .
\end{aligned}
$$

Hence,

$$
\left\|\tau_{n+1}\right\| \geq\left\|\tau_{n+1}(y)\right\|>\left\|\tau_{n}^{\prime}\right\| \geq\left\|\tau_{n}\right\|
$$

This completes the proof.

The last assertion of the theorem is essentially due to Smith [16: Theorem 2.10] in which the assertion is proved for $B=M_{n}$. In case of general subhomogeneous $C^{*}$-algebras we proceed as follows. Let $\tau$ be a bounded linear map from any $C^{*}$-algebra $A$ to $B$ and consider the multiplicity map $\tau_{k}$ from $A \otimes M_{k}$ to $B \otimes M_{k}$. We have then

$$
\left\|\tau_{k}(x)\right\|=\sup \left\|\left[\tau_{k}(x)\right]_{p}\right\|
$$

where $\left[\tau_{k}(x)\right]_{p}$ denotes the image of $\tau_{k}(x)$ in the quotient $C^{*}$-algebra $B \otimes M_{k} / P$ by a primitive ideal $P$ and the supremum is ranging over all primitive ideals. We recall here that the ideal $P$ is written as $I \otimes M_{k}$ for a primitive ideal $I$ of $B$ and the algebra $B \otimes M_{k} / P$ is canonically identified with $B / I \otimes M_{k}$, which may be regarded as a subalgebra of $M_{n} \otimes M_{k}$ (cf. [19]). In these identifications, $\left[\tau_{k}(x)\right]_{p}$ is written as $\pi \otimes 1_{k} \circ \tau_{k}(x)=(\pi \circ \tau)_{k}(x)$ where $\pi$ is the quotient homomorphism: $B \rightarrow B / I \subset M_{n}$. Hence

$$
\left\|\left[\tau_{k}(x)\right]_{p}\right\| \leq\left\|(\pi \circ \tau)_{k}\right\|\|x\| \leq\left\|(\pi \circ \tau)_{n}\right\|\|x\| \leq\left\|\tau_{n}\right\|\|x\|
$$

and $\left\|\tau_{k}\right\| \leq\left\|\tau_{n}\right\|$ for every $k$. Thus we have that $\left\|\tau_{n}\right\|=\|\tau\|_{c b}$. This completes all proofs.

The next Corollary generalizes Proposition 3.1 (a) to arbitrary $n$.

Corollary 3.3. Let $B$ be a $C^{*}$-algebra. Then every bounded linear map $\tau$ from an arbitrary $C^{*}$-algebra $A$ to $B$ is completely bounded and $\left\|\tau_{n}\right\|=\|\tau\|_{c b}$ for a fixed $n$ if and only if $B$ is subhomogeneous with bounded degree $n$.

The above results should be compared with the case of completely positive maps in [20: Theorem 1.2]. We do not have corresponding generalization for the part (b). Precise relations between the dimension of $A$ and the condition 
$\left\|\tau_{n}\right\|=\|\tau\|_{c b}$ for every $\tau$ is not known.

We close this section with an estimate of the index $k_{n}(A)$ in connection with Lemma 2.2 .

Proposition 3.4. Suppose that a $C^{*}$-algebra $A$ has an irreducible representation $\pi$ with $\operatorname{dim} \pi \geq n$, then $k_{n}(A)=n$ and the supremum is attained.

Proof. The inequality $k_{n}(A) \leq n$ follows from [16: Theorem 2.10] because in this case we have that $\|\tau\|_{c b}=\left\|\tau_{n}\right\| \leq n\|\tau\|$. For the other estimate let $p$ be a projection to an $n$-dimensional subspace of the representation space $H_{\pi}$. We identify the algebra $B\left(p H_{\pi}\right)=p B\left(H_{\pi}\right) p$ with $M_{n}$. We have then a contractive completely positive map $\sigma$ from $A$ to $M_{n}$ defined by $\sigma(x)=p \pi(x) p$. We assert that the composition map $\tau=\theta(n) \circ \sigma$ has its completely bounded norm $n$. In fact, let $H_{n}$ be an $n$-dimensional Hilbert space, then the multiplicity representation $\pi \otimes 1_{n}$ is an irreducible representation on the space $H_{\pi} \otimes H_{n}$. Let $\left[e_{j i}\right]$ be a block matrix where $\left\{e_{i j}\right\}$ is a matrix units in $B\left(p H_{\pi}\right)=M_{n}$ and we regard the matrix $\left[e_{j i}\right]$ as an operator on $H_{\pi} \otimes H_{n}$, which is a selfadjoint partial isometry. Since $p \otimes 1_{n}$ is a projection of finite rank, by Kadison's transitivity theorem [17; II Theorem 4.18], there exists for every $\varepsilon>0$ an element $x \in A \otimes M_{n}$ with $\|x\| \leq 1+\varepsilon$ such that

$$
\sigma_{n}(x)=p \otimes 1_{n}\left(\pi \otimes 1_{n}\right)(x) p \otimes 1_{n}=\left[e_{j i}\right] .
$$

Therefore,

$$
n=\left\|\left[e_{i j}\right]\right\|=\left\|(\theta(n) \cdot \sigma)_{n}(x)\right\| \leq\|\tau\|_{c b}(1+\varepsilon),
$$

and hence $\|\tau\|_{c b}=n$. This completes the proof.

\section{§4. Completely Positive Decompositions of Completely Bounded Maps}

Since the concept of complete boundedness is nonselfadjoint counterpart of the concept of complete positivity, it may be natural to think of the question whether every completely bounded map is a linear combination of completely positive maps. In a commutative $C^{*}$-algebra, this happens to coincide with the question whether every bounded linear map is a linear combination of positive maps and the problem of this type has been discussed for years ([13], [22]). It is however only recent days when we have exactly recognized examples of those maps which do not admit decompositions into positive maps in our familiar spaces such as $C[0,1], C_{0}(R), C(T)$ etc. (see the last part of this section). 
We say that a completely bounded (resp. bounded) linear map $\tau$ between $C^{*}$-algebras $A$ and $B$ admits a completely positive (resp. positive) decomposition if $\tau$ is written as a linear combination of completely positive (resp. positive) maps. Moreover we say that a completely positive (resp. positive) decomposition of the map $\tau$ is subject to the norm condition if $\left\|\tau_{i}\right\| \leq\|\tau\|_{c b}\left(\right.$ resp. $\left.\left\|\tau_{i}\right\| \leq\|\tau\|\right)$ in the decomposition $\tau=\tau_{1}-\tau_{2}+i\left(\tau_{3}-\tau_{4}\right)$. Now if we do not restrict the range algebra $B$ there is a result of Loebl [13: Theorem 2.2] which states that even for the space $C(X)$ of continuous functions for an infinite compact Hausdorff space $X$ one can find a bounded selfadjoint linear map $\tau$ from $C(X)$ into the algebra $K(H)$ without having a positive decomposition. Thus again the problem of decomposition is mainly concerned with the properties of $C^{*}$-algebras as the ranges of the maps.

Let $C(\Omega)$ be the algebra of all continuous functions on a Stonean space $\Omega$. It was then known by Tsui [22: Theorem 1.2.10] that every bounded linear map $\tau$ of a $C^{*}$-algebra $A$ into $C(\Omega)$ admits a positive decomposition subject to the norm condition. On the other hand one can easily verify that every bounded (=completely bounded) linear map $\tau$ of $M_{n}$ to $M_{n}$ admits a completely positive decomposition. For in this case $\tau$ is completely determined by the block matrix $a_{\tau}=\left[\tau\left(e_{i j}\right)\right]$ for the matrix units $\left\{e_{i j}\right\}$ of $M_{n}$ and since $\tau$ is completely positive if and only if $a_{\tau}$ is positive, a decomposition of $a_{\tau}$ into a linear combination of positive matrices give rise to a completely positive decomposition of $\tau$. One may then further think of the possibility of completely positive decompositions for a finite von Neumann algebra. In this direction Wittstock [25] has recently proved the following non-commutative version of Tsui's result (actually his result is a little more general, treating module maps).

Theorem 4.1 ([25: Satz 4.5]). Let $B$ be an injective $C^{*}$-algebra, then every completely bounded linear map $\tau$ from a $C^{*}$-algebra $A$ to $B$ admits a completely positive decomposition subject to the norm condition.

Wittstock's proof is based on the investigation of matricial structure of operator algebras through the concept of matrix-sublinear functionals and their extension theorem of Hahn-Banach type. Since his method as well as his results seems to be important for further analysis of the structure of operator algebras we shall introduce the notion of matrix-sublinear functionals. For simplicity we concentrate to $C^{*}$-algebras apart from general matricially ordered *-vector spaces. Let $A$ and $B$ be $C^{*}$-algebras. 
Definition 4.2. A matrix-sublinear functional $\theta: A \rightarrow B$ is a family $\theta=$ $\left(\theta_{n}\right)_{n \in N}$ of maps $\theta_{n}$ of $M_{n}(A)_{h}$ to the family of subsets of $M_{n}(B)_{h}$ satisfying the following conditions:

(i) $\theta_{n}(a) \neq \phi$,

(ii) $\theta_{n}(a+b) \precsim \theta_{n}(a)+\theta_{n}(b)$,

(iii) $0 \precsim \theta_{n}(0)$,

(iv) $\theta_{m}\left(\gamma^{*} a \gamma\right) \precsim \gamma^{*} \theta_{n}(a) \gamma$

for every $a, b \in M_{n}(A)_{h}, \gamma \in M_{n, m}$.

Here the relation $S_{1} \precsim S_{2}$ for two subsets $S_{1}$ and $S_{2}$ in $M_{n}(B)_{h}$ means that for any element $b \in S_{2}$ there exists an element $a$ in $S_{1}$ such that $a \leq b$. The space $M_{n, m}$ means the set of all matrices of type $(n, m)$. A selfadjoint linear map $\tau$ from $A$ to $B$ can be regarded as a matrix-sublinear functional if we consider the family $\left(\tau_{n}\right)_{n \in N}$ of the multiplicity maps of $\tau$. We write $\theta_{1} \precsim \theta_{2}$ for two matrixsublinear functionals $\theta_{1}=\left(\theta_{1 n}\right)$ and $\theta_{2}=\left(\theta_{2 n}\right)$ if $\theta_{1 n}(a) \precsim \theta_{2 n}(a)$ for every $a \in$ $M_{n}(A)_{h}$ and every $n$. Then a key point of the proof of the theorem is provided by the following:

Proposition 4.3 ([25: Corollary 2.2.4]). Let $B$ be an injective $C^{*}$-algebra and $\theta=\left(\theta_{n}\right) ; A \rightarrow B$ be a matrix-sublinear functional. Then there exists a selfadjoint linear map $\tau ; A \rightarrow B$ with $\tau \precsim \theta$.

The readers may recognize the rôle of matrix-sublinear functionals in the following last step of the proof of the theorem.

Let $\tau$ be a selfadjoint completely bounded map from $A$ to $B$. Assume at first that $A$ is unital and define the map $\theta_{n}$ of $M_{n}(A)_{h}$ to the family of subsets of $M_{n}(B)_{h}$ by

$$
\theta_{n}(a)=\left\{\lambda\|\tau\|_{c b}+\tau_{n}(v-w) \mid a \leq \lambda-v-w, \lambda \in R, v, w \in M_{n}(A)_{+}\right\} .
$$

It can be shown that $\theta=\left(\theta_{n}\right)_{n \in N}$ is matrix-sublinear, and by the above Proposition there exists a selfadjoint linear map $\psi: A \rightarrow B$ with $\psi \precsim \theta$. Now for a negative element $a$ of $M_{n}(A)$ put $\lambda=0, v=0$ and $w=-a$ or $\lambda=0, v=-a$ and $w=0$, then we have that $\psi_{n}(a) \leq \pm \tau_{n}(a)$. Hence, $\psi_{n}(a) \leq 0$ and moreover $\left(\psi_{n} \pm \tau_{n}\right)(a) \leq 0$. Thus $\psi$ and $\psi \pm \tau$ are completely positive and we obtain a completely positive decomposition $\tau=1 / 2(\psi+\tau)-1 / 2(\psi-\tau)$. Furthermore, since $\psi\left(1_{A}\right) \leq\|\tau\|_{c b} \circ 1_{B}$ the decomposition satisfies the norm condition.

When $A$ is not unital we simply consider the composition map $\varepsilon \circ \tilde{\tau}$ of the second transpose map $\tilde{\tau}: \tilde{A} \rightarrow \widetilde{B}(\tilde{A}, \widetilde{B}$ are enveloping von Neumann algebras of $A$ and $B$ ) and a projection of norm one from $\widetilde{B}$ to $B$. A completely positive decomposition of the map $\varepsilon \cdot \tilde{\tau}$ implies then a decomposition of $\tau$ with the norm 
condition as well.

Although the above method is interesting this proof is somewhat complicated for the proof itself and a direct proof is given by Paulsen [14]. Let us briefly follow his arguments. Let $A$ be a unital $C^{*}$-algebra and $\tau$ be a selfadjoint complete contraction from $A$ to $B(H)$. Consider the subspace

$$
S=\left\{\left[\begin{array}{ll}
r & a \\
a^{*} & s
\end{array}\right] \mid r, s \in R, a \in A\right\}
$$

and define the map $\phi: S \rightarrow\left(B(H) \otimes M_{2}\right)_{h}$ by

$$
\phi\left[\begin{array}{ll}
r & a \\
a^{*} & s
\end{array}\right]=\left[\begin{array}{cc}
r & \tau(a) \\
\tau(a)^{*} & s
\end{array}\right]
$$

which becomes a unital (real) complete contraction. By Arveson's extension theorems [2: Proposition 1.2.8 and Theorem 1.2.9] $\phi$ possesses not only a completely positive extension to the selfadjoint subspace of $A \otimes M_{2}$ generated by $S$ but also further completely positive extension $\hat{\phi}$ to $A \otimes M_{2}$. We can write the $\operatorname{map} \hat{\phi}$ as

$$
\hat{\phi}\left[\begin{array}{ll}
a & b \\
c & d
\end{array}\right]=\left[\begin{array}{cc}
\phi_{1}(a) & \tau(b) \\
\tau(c) & \phi_{2}(d)
\end{array}\right] .
$$

Put $\psi=1 / 2\left(\phi_{1}+\phi_{2}\right)$. One can then verify that $\psi$ is a unital completely positive map from $A$ to $B(H)$ such that both maps $\psi \pm \tau$ are also completely positive. This completes the case $B(H)$, and the same proof is available for an injective $C^{*}$-algebra.

We remark that an essential part of the theorem is in the case of the algebra $M_{n}$. In fact, if we know that a map $\tau: A \rightarrow M_{n}$ admits a completely positive decomposition with the norm condition we can reduce the case $B(H)$ to its finite dimensional matrix subalgebras and the rest can be done simply by taking cluster points of those decomposed completely positive components because the bounded parts of $L(A, B(H))$ are compact with respect to the $B W^{*}$-topology (cf. [2]). Thus it may be interesting to know an elementary proof of the theorem in the case $B=M_{n}$.

Now in both proofs the completely positive map $\psi$ such that $\psi \pm \tau$ are completely positive is chosen to have the same norm as that of $\tau$. Paulsen [15: Theorem 2.6] has given a stronger version of this fact in which for an arbitrary completely bounded map $\tau$ from $A$ to $B(H)$ (hence for an injective $C^{*}$-algebra) we have a completely positive map $\psi: A \rightarrow B(H)$ with $\|\psi\|=\|\tau\|_{c b}$ such that $\psi \pm$ 
$\operatorname{Re}(\tau)$ and $\psi \pm \operatorname{Im}(\tau)$ are completely positive. Moreover the norm preserving extension theorem of Hahn-Banach type for a completely bounded map from a subspace $S$ of a $C^{*}$-algebra to $B(H)$ is also obtained in [15] and Wittstock [26].

In these circumstances, $T$. Takasaki has given the following general estimate making use of the arguments in [14], [15]. We include here his proof.

Proposition 4.4. Let $A$ and $B$ be $C^{*}$-algebras and $\tau$ be a selfadjoint completely bounded map of $A$ to $B$. Let $\psi$ be a completely positive map of $A$ to $B$ such that both maps $\psi \pm \tau$ are completely positive, then $\|\tau\|_{c b} \leq\|\psi\|$.

Proof. Assume first that both $A$ and $B$ are unital. Let $\phi$ be a map from $M_{2}(A)$ to $M_{2}(B)$ defined as:

$$
\phi\left[\begin{array}{ll}
a & b \\
c & d
\end{array}\right]=\left[\begin{array}{cc}
\psi(a) & \tau(b) \\
\tau(c) & \psi(d)
\end{array}\right] .
$$

We assert that $\phi$ is completely positive. Indeed, let

$$
\psi+\tau=U^{*} \pi_{1}(\cdot) U, \quad \psi-\tau=V^{*} \pi_{2}(\cdot) V
$$

be the Stinespring representations of completely positive maps $\psi \pm \tau$ ([17: IV, Theorem 3.6]). Then we have

$$
\begin{aligned}
{\left[\begin{array}{ll}
\psi(a) & \tau(b) \\
\tau(c) & \psi(d)
\end{array}\right]=} & \left.\frac{1}{2}\left\{\begin{array}{cc}
U^{*} \pi_{1}(a) U & U^{*} \pi_{1}(b) U \\
U^{*} \pi_{1}(c) U & U^{*} \pi_{1}(d) U
\end{array}\right]+\left[\begin{array}{cc}
V^{*} \pi_{2}(a) V & -V^{*} \pi_{2}(b) V \\
-V^{*} \pi_{2}(c) V & V^{*} \pi_{2}(d) V
\end{array}\right]\right\} \\
= & \frac{1}{2}\left[\begin{array}{cc}
U^{*} & 0 \\
0 & U^{*}
\end{array}\right] \pi_{1} \otimes 1_{2}\left(\left[\begin{array}{ll}
a & b \\
c & d
\end{array}\right]\right)\left[\begin{array}{cc}
U & 0 \\
0 & U
\end{array}\right] \\
& +\frac{1}{2}\left[\begin{array}{cc}
V^{*} & 0 \\
0 & -V^{*}
\end{array}\right] \pi_{2} \otimes 1_{2}\left(\left[\begin{array}{ll}
a & b \\
c & d
\end{array}\right]\right)\left[\begin{array}{cc}
V & 0 \\
0 & -V
\end{array}\right]
\end{aligned}
$$

which shows the assertion. Next take an element $x$ of $M_{n}(A)$, then re-shuffling the matrix $\left[\begin{array}{ll}0 & x \\ x^{*} & 0\end{array}\right]$ of $M_{2}\left(M_{n}(A)\right)=M_{n}\left(M_{2}(A)\right)$, we can see that

$$
\phi_{n}\left[\begin{array}{ll}
0 & x \\
x^{*} & 0
\end{array}\right]=\left[\begin{array}{cc}
0 & \tau_{n}(x) \\
\tau_{n}(x)^{*} & 0
\end{array}\right] .
$$

Hence,

$$
\left\|\tau_{n}(x)\right\|=\left\|\phi_{n}\left[\begin{array}{ll}
0 & x \\
x^{*} & 0
\end{array}\right]\right\| \leq\left\|\phi_{n}\right\|\|x\| .
$$

On the other hand, since $\phi$ is completely positive we have

$$
\left\|\phi_{n}\right\|=\|\phi\|=\|\phi(1)\|=\|\psi(1)\|=\|\psi\| .
$$


Therefore, $\left\|\tau_{n}\right\| \leq\|\psi\|$ for every $n$ and $\|\tau\|_{c b} \leq\|\psi\|$.

For nonunital case, consider the double transposes $\tilde{\tau}$ and $\tilde{\psi}$ of $\tau$ and $\psi$ as the maps between the enveloping von Neumann algebras $\widetilde{A}$ and $\widetilde{B}$. We have then the same assumption for $\tilde{\tau}$ and $\tilde{\psi}$, and $\|\tilde{\tau}\|_{c b} \leq\|\tilde{\psi}\|$. It suffices then to note that $\|\tau\|_{c b}=\|\tilde{\tau}\|_{c b}$ and $\|\psi\|=\|\tilde{\psi}\|$.

One might suspect the theorem of completely positive decompositions within the category of von Neumann algebras. This is however not the case. There exists a completely bounded map from a $C^{*}$-algebra to certain von Neumann algebra which does not admit a completely positive decomposition [10: Example 12]. The author suspects that the converse of the Theorem 4.1 would be true. Namely we propose the following conjecture.

Let $B$ be a unital $C^{*}$-algebra and suppose that for any $C^{*}$-algebra $A$ every completely bounded map from $A$ to $B$ admits a completely positive decomposition. Then $B$ is an injective $C^{*}$-algebra.

The answer to this question will show another aspect of injectivity but as of now we have few informations about reasonable existence of completely bounded maps with no completely positive decomposition for nonseparable $C^{*}$-algebras, camparing with discussions below for separable cases. Thus even for commutative $C^{*}$-algebras the conjecture has been remained open.

For a separable $C^{*}$-algebra the answer to the conjecture has been recently given by Huruya in [8: Corollary 7]. Combining this with the previous result by Huruya and the author [10: Theorem 11] we have now the following result.

Theorem 4.5. Let $B$ be a separable $C^{*}$-algebra. The following assertions are equivalent:

(a) For any $C^{*}$-algebra $A$, every completely bounded map $\tau$ of $A$ to $B$ admits a completely positive decomposition.

(b) With the same notations, the map $\tau$ admits a completely positive decomposition subject to the norm condition.

(c) For any $C^{*}$-algebra $A$, every bounded linear map $\tau$ of $A$ to $B$ admits a positive decomposition.

(d) $B$ is finite dimensional (injective).

The result is somewhat unsatisfactory because aspects of injectivity are essentially expressed in nonseparability.

We remark that in the above theorem each of the conditions makes the 
algebra $B$ unital. For instance, assume the condition (a) and that $A$ is acting on the space $H$ nondegenerately. Since $B$ is separable, by [17: I, Remark 7.6] there exists a commuting countable approximate identity $\left\{u_{n}\right\}$ for $B$. Let $C$ be a commutative $C^{*}$-subalgebra generated by $\left\{u_{n}\right\}$ and let $A$ be a $C^{*}$-algebra generated by $C$ and the identity operator 1 . Then $C$ is a closed ideal of $A$ and every element of $A$ is written as $x+\lambda 1$. Let $\tau$ be the bounded selfadjoint projection map: $x+\lambda 1 \rightarrow x$ considered as a map from $A$ to $B$. The map $\tau$ is completely bounded by Proposition 3.1 (a). Suppose $\tau$ be majorized by a completely positive map $\phi$ of $A$ to $B$ such that $\phi-\tau$ is also completely positive. Then we have

$$
u_{n}=\tau\left(u_{n}\right) \leq \phi\left(u_{n}\right) \leq \phi(1)
$$

for every $n$, and since $u_{n}$ converges strongly to the identity operator we have that $1 \leq \phi(1)$. Hence $\phi(1)$ is invertible in $B$ and $B$ is unital. On the other hand it is well known that a finite dimensional $C^{*}$-algebra must be unital.

Now as in Section 2 we have to discuss the existence of completely bounded maps without completely positive decompositions as counterparts of the theorem. This will be done by factorization maps through certain commutative $C^{*}$-algebras. Let $\alpha N$ be the space of one-point compactification of the set of natural integers. We consider the infinite copies $\left\{\Gamma_{n}=\left(x_{n 1}, x_{n 2}, \ldots, x_{n m}, \ldots, x_{n \infty}\right)\right\}$ of $\alpha N$ and let $\Gamma$ be the one-point compactification of their topological sum. The following two lemmas in [8] play the rôle corresponding to Lemmas 2.2 and 2.3.

Lemma 4.6. Let $A$ be a separable unital $C^{*}$-algebra in which there exists a selfadjoint element with the spectrum of infinite limits points. Then there exist unital completely positive maps $\rho_{A}: C(\Gamma) \rightarrow A$ and $\sigma_{A}: A \rightarrow C(\Gamma)$ such that $\sigma_{A^{\circ}}^{\circ} \rho_{A}=$ identity.

On the other hand, by using an element with the infinite spectrum we have:

Lemma 4.7. Let $B$ be an infinite dimensional separable unital $C^{*}$-algebra, then there exist unital completely positive maps $\mu_{B}: C(\alpha N) \rightarrow B$ and $v_{B}: B \rightarrow$ $C(\alpha N)$ such that $v_{B} \circ \mu_{B}=$ identity.

With these lemmas the problem is reduced to that of the maps between $C(\Gamma)$ and $C(\alpha N)$. Now, we regard $\alpha N$ as the one-point compactification of double sequence $\left(y_{n m}\right)$ denoted by $Y_{0}$. There is then an old example of a selfadjoint map from $C(\Gamma)$ to $C\left(Y_{0}\right)$ due to Tsui [22] and Kaplan [11], which is defined as 


$$
\tau(f)\left(y_{n, m}\right)=f\left(x_{n, m}\right)-f\left(x_{n, m+1}\right), \quad \tau(f)\left(y_{\infty}\right)=0 .
$$

The map does not admit positive decompositions. In order to find a selfadjoint map whose positive decompositions are not subject to the norm condition, we use a map $\tau_{k}$ defined for a positive integer $k$ as follows:

$$
\begin{array}{ll}
\tau_{k}(f)\left(y_{n, m}\right)=1 / 2\left(f\left(x_{n, m}\right)-f\left(x_{n, m+1}\right)\right) & \text { if } n \leq k, \\
\tau_{k}(f)\left(y_{n, m}\right)=0 & \text { if } n>k, \\
\tau_{k}(f)\left(y_{\infty}\right)=0 . &
\end{array}
$$

It can be shown that $\left\|\tau_{k}\right\|=1$ and for every positive decomposition $\tau_{k}=\tau_{k}^{+}-\tau_{k}^{-}$ we have

$$
\left\|\tau_{k}^{+}\right\| \geq k / 2 \text { and }\left\|\tau_{k}^{-}\right\| \geq k / 2 .
$$

It would be better to reformulate here Huruya's existence theorem [8: Theorem 6] in the following form.

Theorem 4.8. Let $A$ be a $C^{*}$-algebra satisfying the condition of Lemma 4.6 and $B$ be an infinite dimensional separable unital $C^{*}$-algebra. Then the subspace of completely bounded maps with completely positive decomposition is nowhere dense in the space $C B(A, B)$. In particular, the subspace for the algebra $A$ is nowhere dense in $C B(A)$.

Indeed, if $\|\psi-\tau\|<1 / 2$ for the map $\tau$ defined above, $\psi$ does not admit a positive decomposition [8: Lemma 3], hence the theorem is true for the space $C B\left(C(\Gamma), C\left(Y_{0}\right)\right)=L\left(C(\Gamma), C\left(Y_{0}\right)\right)$. Therefore it must be also true for the space $C B(A, B)$ by the above factorization maps. The result also means that the subspace of positively decomposable maps as well as that of maps with completely positive decompositions is nowhere dense in the space $L(A, B)$.

Comparing with these results main difficulty in nonseparable cases lies in the point that we lack suitable lifting theorem for positive and completely positive maps.

As we have mentioned before, a derivation in a $C^{*}$-algebra is completely bounded and if it is inner a polarization formula shows that it has a completely positive decomposition. Thus it will be interesting to know the condition of algebras for which every derivation has a completely positive decomposition.

So far we have seen that most problems about completely positive decompositions can be reduced to commutative cases. We shall now turn to the problem for the maps between commutative $C^{*}$-algebras, in particular on the same domain and range. We recall that in commutative cases the complete positivity 
coincides with the positivity by Stinespring's theorem and the complete boundedness coincides with the boundedness. Thus the problem could well be said to be of the type of (generalized) Hahn decompositions. Note first that the decomposability of the maps on the space $C(X)$ does not characterize the space $X$ as a Stonean space. In fact, one can verify that every bounded linear map on $C(\alpha N)$ admits a Hahn decomposition (positive decomposition). Loebl [13] introduced before the variation of a map to study Hahn decompositions as a generalization of the variation of a measure, but this concept was not so effective for the problem.

Now suppose that a compact space $X$ has the property such that the space $C(X)$ satisfies the conditions in Lemmas 4.6 and 4.7. We see then by Theorem 4.8 that the subspace of bounded linear maps with positive decompositions is nowhere dense in the space $L(C(X))$. A good example of such a space is an uncountable metric space such as closed intervals and torus (cf. also [10]). Thus even in those spaces such as $C[0,1]$ and $C(T)$ subspaces of positively decomposable maps are quite thin in the whole spaces of bounded linear maps.

As we have seen before, even the simple space $C(\alpha N)$ can not be an algebra of ranges for positive decompositions. Moreover, since $\alpha N$ can be embedded into the space $X$ which contains a convergent sequence of distinct points, the lifting theorem of Borsk-Dugundgi [3: Theorem 3.11] implies that spaces having this more weak property can not be the range algebras for positive decompositions either.

\section{§5. Concluding Remarks}

In the previous Section 4 we have discussed the possibility of completely positive decompositions together with the norm condition. In case of a selfadjoint functional $\varphi$ on a $C^{*}$-algebra however more stronger decomposition is known: namely there exists a unique positive decomposition $\varphi=\varphi^{+}-\varphi^{-}$such that $\|\varphi\|=\left\|\varphi^{+}\right\|+\left\|\varphi^{-}\right\|$. There are some cases in which we can find this type of (so-called) minimal decomposition.

Proposition 5.1. Let $A$ be a unital $C^{*-a l g e b r a}$ and $B$ be an injective $C^{*-}$ algebra. Then every unital selfadjoint completely bounded map $\tau$ of $A$ to $B$ has a minimal decomposition.

Proof. Take a completely positive map $\psi: A \rightarrow B$ with $\|\psi\|=\|\tau\|_{c b}$ such that 
$\psi \pm \tau$ are completely positive. Then

$$
\|\psi+\tau\|=\|\psi(1)+1\|=\|\psi\|+1
$$

and since $\psi(1) \geq 1$ we also have

$$
\|\psi-\tau\|=\|\psi(1)-1\|=\|\psi(1)\|-1=\|\psi\|-1 .
$$

Hence for a completely positive decomposition

$$
\tau=1 / 2(\psi+\tau)-1 / 2(\psi-\tau)
$$

we have

$$
\|\tau\|_{c b}=1 / 2(\|\psi\|+1)+1 / 2(\|\psi\|-1)=\|1 / 2(\psi+\tau)\|+\|1 / 2(\psi-\tau)\| .
$$

We remark that the above proposition is valid if $\tau\left(1_{A}\right)=\lambda 1_{B}$.

The map $\theta(n)$ has a minimal decomposition and the decomposition is unique ([8]). There are other examples of maps in $M_{n}$ which have unique minimal decompositions. But in general we can not expect neither this type of decomposition nor the uniqueness even in matrix algebras. Other reasonable conditions to ensure the existence of the minimal decomposition is not known.

The problem of polar decompositions of completely bounded maps seems to be along similar lines as in the case of minimal decompositions in relation with the case of functionals. Results known in this direction are only those results about bounded maps proved in Tsui [22]. We may not expect much about polar decompositions but more investigations should be done for completely bounded maps.

We finally review the duality of algebras (or groups) proposed by Walter [23] in the category of completely bounded maps.

Let $A$ and $B$ be Banach algebras with (possibly commutative) two involutions one of which must be isometric. An involution: $a \rightarrow \bar{a}$ is said to be commutative if $(a b)^{-}=\bar{a} \bar{b}$ instead of $\bar{b} \bar{a}$.

Definition 5.2. The algebras $A$ and $B$ are said to be dual in the first sense if the following two conditions are satisfied:

(1) There exist Banach algebra homomorphisms $i_{A}, i_{B}$ from $A, B$ onto dense subalgebras of $C^{*}$-algebras $C^{*}(A), C^{*}(B)$ respectively. The maps $i_{A}$ and $i_{B}$ are one-to-one and preserve an involution on $A$ and $B$ respectively.

(2) There exist isometric Banach algebra isomorphisms $j_{A}, j_{B}$ from $A, B$ to the algebras $C B\left(C^{*}(B)\right), C B\left(C^{*}(A)\right)$ respectively and they preserve an involution on each $A$ and $B$. 
Definition 5.3. The algebras $A$ and $B$ are said to be dual in the second sense if the following two conditions are satisfied:

(1) The same condition as in (1) in the Definition 5.2.

(2) There exist Banach space isometries (into) $O_{A}: A \rightarrow C^{*}(B)^{*}$ and $O_{B}$ : $B \rightarrow C^{*}(A)^{*}$. They preserve an involution on each $A$ and $B$ respectively.

Now let $G$ be a locally compact group and set $A=L^{1}(G)$ with (isometric) involutions:

$$
f \longrightarrow f^{\#}=\Delta^{-1} f^{b} \text { and } f \longrightarrow \bar{f}
$$

where $\Delta$ is the modular function of $G$ and $f^{b}(x)=\bar{f}\left(x^{-1}\right)$. Let $B=A(G)$ (Fourier algebra of $G$ ) with (isometric) involutions:

$$
a \longrightarrow \bar{a} \text { and } a \longrightarrow a^{b} .
$$

Assuming $C^{*}(A)=C^{*}(G)$ (group $C^{*}$-algebra) and $C^{*}(B)=C_{0}(G)$, Walter has proved in [23] that they are dual in the first sense. This pair of algebras has been considered to involve the duality of the group $G$ in the duality theory of a noncommutative locally compact group.

Let $A$ and $B$ be again the algebras $L^{1}(G)$ and $A(G)$ with the same involutions. Then as before condition (1) of Definition 5.3 holds and condition (2) holds with involutions $f \rightarrow \bar{f}$ on $L^{1}(G)$ and $a \rightarrow a^{b}$ on $A(G)$ since

$$
L^{1}(G) \subseteq M^{1}(G)=C_{0}(G)^{*}=C^{*}(A(G))^{*}
$$

and

$$
A(G) \subseteq B(G)=C^{*}(G)^{*}=C^{*}\left(L^{1}(G)\right)^{*}
$$

where $B(G)$ denotes the Fourier-Stieltjes algebra of $G$. Thus, $L^{1}(G)$ and $A(G)$ are also dual in the second sense.

An advantage of this duality theory is in the point that it is also applicable to other non-group related algebras. For instance, let $A=\left(M_{n},{ }^{*},\|\circ\|_{I, r}\right)$, the $n \times n$ matrices with the matrix product and the norm $\left\|\left[a_{i j}\right]\right\|_{I, r}=\sup _{i} \sum_{j=1}^{n}\left|a_{i j}\right|$ and let $B=\left(M_{n}, \circ,\|\circ\|_{\infty}\right)$, the $n \times n$ matrices with the Schur product and the norm $\left\|\left[a_{i j}\right]\right\|_{\infty}=\sup _{i, j}\left|a_{i j}\right|$. Then it can be shown that $A$ and $B$ are dual in the first sense. In this way one can reach the unified understanding about dualities of these seemingly unrelated cases. 


\section{References}

[1] Ando, T., Completely contractive map, Seminar note, 1977.

[2] Arveson, W. B., Subalgebras of $C^{*}$-algebras, Acta Math., 123 (1969), 141-224.

[3] Bade, W. G., The Banach space $C(S)$, Lecture note 26, Århus, 1971.

[4] Choi, M. D. and Effros, E. G., Nuclear $C^{*}$-algebras and the approximation property, Amer. J. Math., 100 (1978), 61-79.

[ 5] Christensen, E., Extensions of derivations II, Math. Scand., 50 (1982), 111-122.

[6] Haagerup, U., Solution of the similarity problem for cyclic representations of $C^{*}$ algebras, preprint.

[ 7] Hadwin, D. W., Dilations and Hahn decompositions for linear maps, Canadian $J$. Math., 33 (1981), 826-839.

[8] Huruya, T., Decompositions of completely bounded maps, preprint.

[9] —- On compact completely bounded maps of $C^{*}$-algebras, preprint.

[10] Huruya, T. and Tomiyama, J., Completely bounded maps of $C^{*}$-algebras, J. Operator Theory, to appear.

[11] Kaplan, S., An example in the space of bounded operators from $C(X)$ to $C(Y)$, Proc. Amer. Math. Soc., 38 (1973), 595-597.

[12] Loebl, R. I., Contractive linear maps on $C^{*}$-algebras, Michigan Math. J., 22 (1975), 361-366.

[13] — A Hahn decomposition for linear maps, Pacific J. Math., 65 (1976), 119133.

[14] Paulsen, V. I., Completely bounded maps on $C^{*}$-algebras and invariant operator ranges, Proc. Amer. Math. Soc., 86 (1982), 91-96.

[15] — Every completely polynomially bounded operator is similar to a contraction, J.Func. Anal., to appear.

[16] Smith, R. R., Completely bounded maps between $C^{*}$-algebras, preprint.

[17] Takesaki, M., Theory of operator algebras I, Springer, 1979.

[18] Takasaki, T. and Tomiyama, J., On the geometry of positive maps in matrix algebras, Math, Zeit., 184 (1983), 101-108.

[19] Tomiyama, J., Applications of Fubini type theorem to the tensor product of $C^{*}$ algebras, Tôhoku Math. J., 19 (1967), 213-226.

[20] — On the difference of $n$-positivity and complete positivity, J. Functional Analysis, 49 (1982), 1-9.

[21] — On the transpose map of matrix algebras, Proc. Amer. Math. Soc., 88 (1983), 635-638.

[22] Tsui, S-K. J., Decompositions of linear maps, Trans. Amer. Math. Soc., 230 (1977), $87-112$.

[23] Walter, M., Dual and dual space algebras, preprint.

[24] Wassermann, S., The slice map problem for $C^{*}$-algebras, Proc. London Math. Soc., 32 (1976), 537-559.

[25] Wittstock, G., Ein operatorwertiger Hahn-Banach Satz, J. Functional Analysis, 40 (1981), 127-150.

[26] — Extension of completely bounded $C^{*}$-module homomorphism, preprint.

Note Added (June, 1983): The author has learned from T. Huruya that the conjecture in Section 4 is negative even in commutative cases. Indeed, Huruya has constructed a com- 
mutative counter example making use of the attached sum of two Stonean spaces with limit points. The example as well as the other results will be published elsewhere. 
